\title{
LISTADO SISTEMÁTICO DE LOS ORIBÁTIDOS (ACARIFORMES, ORIBATIDA) DEL NOROESTE DE LA PENÍNSULA IBÉRICA
}

\author{
L. S. Subías (*) y M. E. Mínguez (*)
}

\begin{abstract}
RESUMEN
En el presente trabajo se ha estudiado una de las zonas de la Península Ibérica menos conocidas desde el punto de vista oribatológico, el noroeste, que comprende toda Galicia, León y el norte de Portugal. Tras recopilar todas las citas de Oribátidos existentes hasta la fecha, 125 en total, se han incorporado 99 nuevas de las que tres: Trhypochthonius tectorum congregator, Moritzoppia oreia y Eupelops subexutus son, a su vez, nuevas citas ibéricas, mientras que otras 9 podrían ser nuevas especies para la ciencia, figurando en el listado como "sp."

Palabras clave: Ácaros oribátidos, faunística, península Ibérica, nuevas citas.
\end{abstract}

\section{ABSTRACT \\ Systematic checklist of the Oribatids (Acariformes, Oribatida) from North- western Iberian Peninsula}

Our knowledge about the oribatids mites fauna from northwestern Iberian Peninsula (Galicia, province of León and northern Portugal) is scarce. Only 125 species were previously recorded from this region. In this paper we add 99 new taxa to this systematic list; three of them are recorded for the first time in the Iberian Peninsula: Trhypochthonius tectorum congregator, Moritzoppia oreia and Eupelops subexutus, and nine of them, labeled as "sp.", are probably new species for Science.

Key words: Oribatid mites, faunistic, Iberian peninsula, new records.

\section{Introducción}

La Península Ibérica, y más concretamente España, ha sido objeto en los últimos 35 años de intensos estudios en lo referente a su fauna oribatológica, y gracias a ello extensas zonas de ella son muy bien conocidas y eso que contamos con un extenso y muy variado territorio con los más diversos ambientes ecológicos.
Una de las pocas zonas que quedaban por estudiar en profundidad era el noroeste ibérico, de gran interés para poder comparar su fauna, de características euroatlánticas, con otras faunas similares así como con la fauna de la región mediterránea, que ocupa la mayor parte de la Península Ibérica. Para ello se ha realizado una revisión previa de todas las citas existentes hasta la fecha en dicha zona, que comprende toda Galicia, la provincia de León y el

* Departamento de Zoología. Facultad de Biología. Universidad Complutense. 28040 Madrid.

E-mail: subias@eucmax.sim.ucm.es 
norte de Portugal, recopilándose un total de 125 citas de distintos ácaros oribátidos. A ellas se han añadido los oribátidos obtenidos en el estudio de 16 muestras de suelo recolectadas en diversas localidades repartidas por toda la zona objeto del trabajo, obteniéndose como consecuencia un listado final que incluye 224 especies diferentes, número elevado, sobre todo si se compara con otras faunas conocidas. Así, por ejemplo se ha publicado recientemente un listado de especies de China (Aoki et al., 1997) en el que se recopilan un total de 312 especies, lo que nos da una idea de lo que queda por conocer en extensas zonas de la Tierra y a su vez lo relativamente completo de este estudio.

\section{Material y métodos}

Para la presentación de los datos obtenidos se ha elaborado una tabla esquemática donde se incluyen, ordenados sistemáticamente, todos los taxones presentes en la región en estudio, así como las localidades en que éstos han aparecido; para ello a continuación de cada especie vienen 20 columnas distribuidas de la siguiente manera: en las seis primeras se han agrupado, básicamente por provincias, las citas previas ya existentes en la bibliografía utilizándose las abreviaturas (en "negrita") de LU (Lugo), LC (La Coruña), OR (Orense), PO (Pontevedra), LN (León) y NP (Norte de Portugal) y señalándose en el interior de la casilla correspondiente, mediante un símbolo, la referencia bibliográfica correspodiente a la primer cita del taxon en cuestión. La simbología utilizada sería la siguiente:

\author{
AS - Arribas, Subías y Ruiz, 1984 \\ G0 - Grandjean, 1928 \\ G1 - Grandjean, 1931 \\ G2 - Grandjean, 1932 \\ G3 - Grandjean, 1935 \\ G4 - Grandjean, 1936 \\ G5 - Grandjean, 1954 \\ G6 - Grandjean, 1956 \\ G7 - Grandjean, 1956b \\ G8 - Grandjean, 1962 \\ M1 - Mihelcic, 1956a \\ M2 - Mihelcic, 1956b \\ M3 - Mihelcic, 1957 \\ M4 - Mihelcic, 1964 \\ MR - Mínguez, Ruiz y Subías, 1985 \\ MS - Mínguez y Subías, 1998 \\ N1 - Niedbala, 1984a \\ N2 - Niedbala, 1984b \\ N3 - Niedbala, 1992 \\ P1 - Pérez-Í́nigo, 1965 \\ P2 - Pérez-Íñigo, 1969a
}

P3 - Pérez-Íñigo, 1969b P4 - Pérez-Íñigo, 1970 P5 - Pérez-Íñigo, 1971 P6 - Pérez-Íñigo. 1972 P7 - Pérez-Íñigo, 1974 P8 - Pérez-Íñigo, 1988 P9 - Pérez-Íñigo, 1993 P0 - Pérez-Íñigo, 1997

PS - Pérez-Íñígo y Subías, 1978 RS - Ruiz y Subías, 1984 S1 - Subías y Arillo, 1998 S2 - Subías y Arillo, (en prensa) S3 - Subías y Rodríguez, 1986a S4 - Subías y Rodríguez, 1986b S5 - Subías y Rodríguez, 1987 S6 - Subías y Rodríguez, 1988 SC - Schubart, 1975

SR - Subías, Rodríguez y Mínguez, 1987 SU - Subías, 1982 TR - Travé, 1957 WO - Woas, 1981
La referencia seguida de una comilla de superíndice quiere decir que existen otras citas posteriores de la misma especie en dicha provincia. Las 14 columnas que siguen a continuación se corresponden con las muestras que han sido estudiadas para este trabajo; vienen designadas por una abreviatura en cursiva y cuando un taxon ha aparecido en la misma se señala con una $X$ el recuadro correspondiente. La relación de dichas muestras es la siguiente:

L1- (Lugo). Los Ancares. 4-X-1983. Musgos y hojarasca de abedul. L. S. Subías leg.

L2- (Lugo). San Román de Cervantes. 4-X-1983. Hojarasca de brezo y pino. L. S. Subías leg.

C1- (La Coruña). Boiro. 15-VIII-1978. Hojarasca de roble. L. S. Subías leg.

C2- (La Coruña). Codeso. 3-X-1983. Hojarasca de helechos. L. S. Subías leg.

O1- (Orense). Suacenza. 10-IV-1976. Hojarasca de roble. R. Outerelo leg.

O2- (Orense). La Canda. 1-X-1983. Musgo en suelo encharcado. E. Ruiz leg.

P1- (Pontevedra). Figueirido. 13-II-1977. Musgos. V. Monsaerrat leg.

P2- (Pontevedra). Poyo. 15-VII-1975. Hojarasca de vegetación costera. J. J. Presa leg.

P3- (Pontevedra). Moscoso. 2-I-1974. Musgos sobre rocas y troncos. R. Outerelo leg.

P4- (Pontevedra). Vilapouca. 3-X-1983. Musgos y hojarasca de roble. E. Ruiz leg.

P5- (Pontevedra). Bayona. 18-V-1998. Musgo de roca y hojarasca de pino y plantas cultivadas. L. S. Subías leg.

P6- (Pontevedra). La Guardia. 16-V-1998. Hojarasca de roble y mimosa. L. S. Subías leg.

LE- (León). Boisán. 6-VI-1997. Hojarasca de vegetación de talud de río. L. S. Subías leg.

MI- (Minho). Geres. 14-VII-1984. Hojarasca de roble. R. Outerelo leg.

\section{Resultados}

Los resultados son básicamente los expuestos en la tabla que viene a continuación, siendo de destacar que tres de los taxones citados, Trhypochthonius tectorum congregator, Moritzoppia oreia y Eupelops subexutus (señalados con un * en la tabla) lo son por primera vez para la Península Ibérica y otros nueve, que figuran como "sp.", muy probablemente sean especies nuevas para la ciencia, mientras que 87 de los 224 taxones recolectados en total, son la primera vez que se citan en la zona en estudio. 


\begin{tabular}{|c|c|c|c|c|c|c|c|c|c|c|}
\hline $\bar{\Sigma}$ & & $x$ & & & & & & & & \\
\hline | & & $\times$ & & & & & & & & \\
\hline$\stackrel{\circ}{\circ}$ & & & & & & & & & & \\
\hline$\stackrel{\leftarrow}{\circ}$ & & & & $\times$ & $\times$ & $\times$ & & & & \\
\hline I & & & & & & & & & & \\
\hline$\varrho^{\infty}$ & & & & & & & & & & \\
\hline$\stackrel{\alpha}{a}$ & & $\times \quad \times$ & & & & & & & & \\
\hline$a^{-}$ & & $\begin{array}{lllllll} & \times & \times & \times\end{array}$ & & & & & & & & \\
\hline ฮ & & & & & & & & & & \\
\hline $\bar{o}$ & & $\begin{array}{lll}x \times & \times & \times\end{array}$ & & & & & & & & \\
\hline 0 & & & & & & & & & & \\
\hline j & & & & & & & & & $\times$ & \\
\hline$\exists$ & & & & & & & & & & \\
\hline$\Xi$ & & & & & & & & & & \\
\hline$\frac{a}{2}$ & & & & & & & & & & $\stackrel{m}{z}$ \\
\hline 3 & $\underset{\infty}{\infty}$ & & $\begin{array}{ll}D & \infty \\
\infty & \end{array}$ & $\begin{array}{l}\infty \\
\mathbb{x}\end{array}$ & & & $\begin{array}{l}\infty \\
\simeq\end{array}$ & $\stackrel{\infty}{\propto}$ & & \\
\hline \& & & & & & $\Sigma$ & & & & & 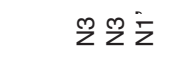 \\
\hline 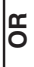 & & & & & & & & & & $\stackrel{m}{z}$ \\
\hline 0 & & & & & & & & & & 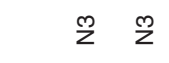 \\
\hline 1 & & & & & & & & & & \\
\hline & 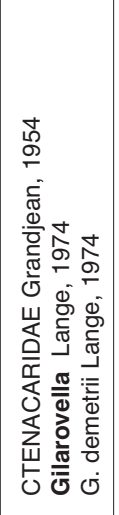 & 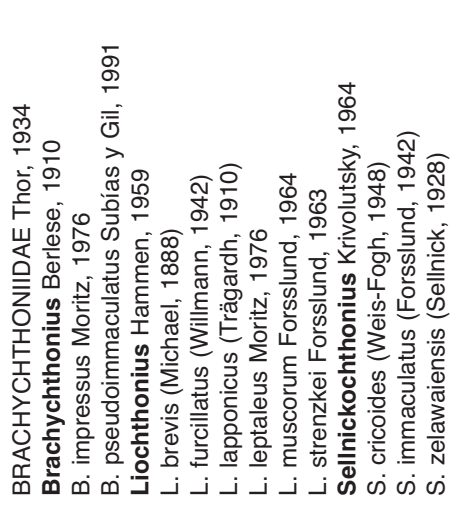 & 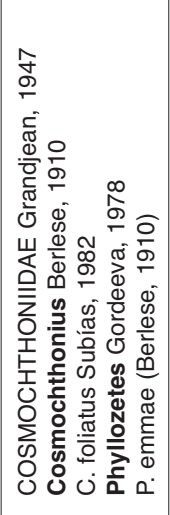 & 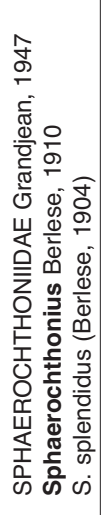 & 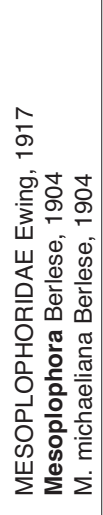 & 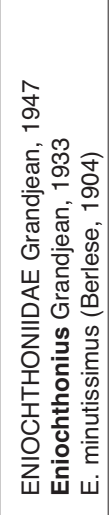 & 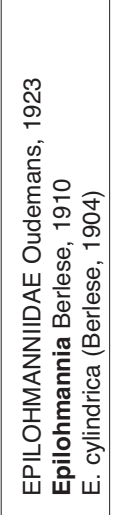 & 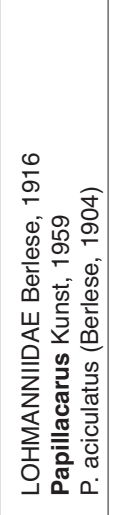 & 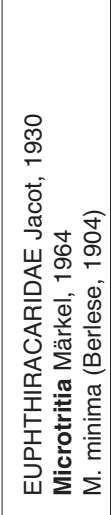 & 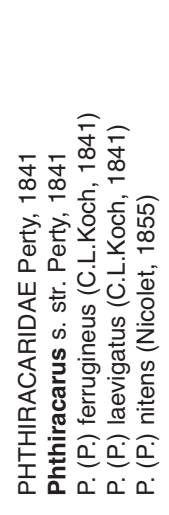 \\
\hline & -1 & NM + thono & 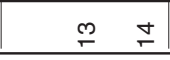 & 요 & $\stackrel{\circ}{\circ}$ & 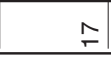 & $\begin{array}{l}\infty \\
\\
\end{array}$ & 익 & ล & $\bar{N} \mathcal{N}$ \\
\hline
\end{tabular}




\begin{tabular}{|c|c|c|c|c|c|c|c|c|c|c|}
\hline $\bar{\Sigma}$ & & $x$ & & & $x$ & & & & & \\
\hline 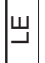 & & & & & & & & & & \\
\hline 0 & & $\times$ & & & & & & & & \\
\hline 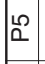 & & & & $\times$ & & $\times$ & & & & \\
\hline$a$ & & & & & & & & & & \\
\hline$\infty$ & & & & & & & & & & \\
\hline$\tilde{\alpha}$ & & & & & & & & & & \\
\hline $\bar{\alpha}$ & & & $\times$ & & & & & & & \\
\hline กิ & & & & & & & & & & \\
\hline 5 & $\times$ & $\times$ & & & & & $\times$ & & & \\
\hline$\widetilde{\mathcal{O}}$ & & & & & & & & & & \\
\hline $\bar{j}$ & & & $\times$ & & & & & $\times$ & & \\
\hline$\Im$ & & & & & & & & & & \\
\hline$\Xi$ & & & & & & & & & & \\
\hline$\hat{\mathbf{z}}$ & $\stackrel{m}{z}$ & $\stackrel{m}{z}$ & & & $\sum$ & & & $\stackrel{2}{\xi}$ & & \\
\hline 3 & $\stackrel{\infty}{\infty}$ & $\tilde{z}$ & 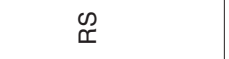 & & & & & & & \\
\hline$\circ$ & $\stackrel{m}{z}$ & $\bar{z} \quad M$ & $\begin{array}{ll}\dot{0} & \varrho\end{array}$ & & & & & œ & व & $\circ$ \\
\hline$\tilde{\alpha}$ & $\stackrel{m}{z}$ & $\stackrel{m}{z}$ & & & & & & & & \\
\hline 9 & $\stackrel{m}{z}$ & $\stackrel{M}{z} \stackrel{m}{z}$ & & & & & & & : & \\
\hline 3 & & & & & & & & & & \\
\hline 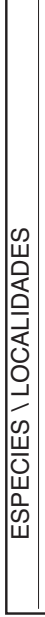 & 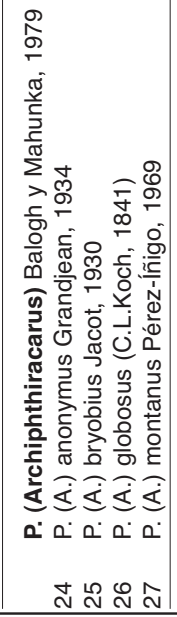 & 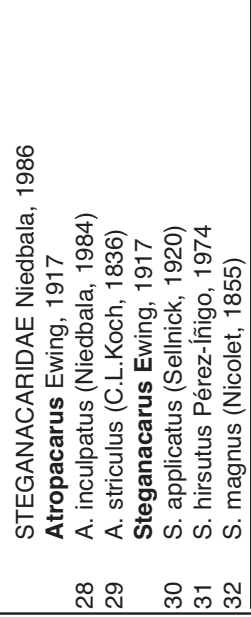 & 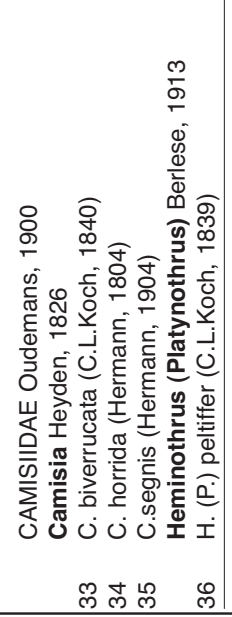 & 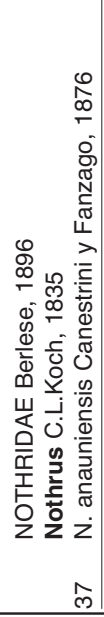 & 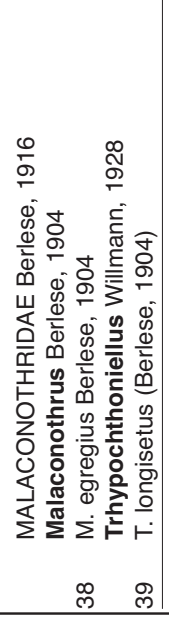 & 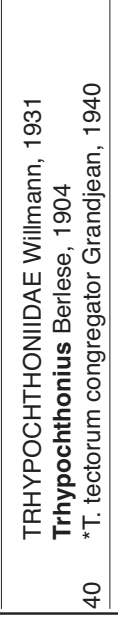 & 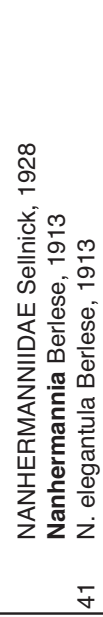 & 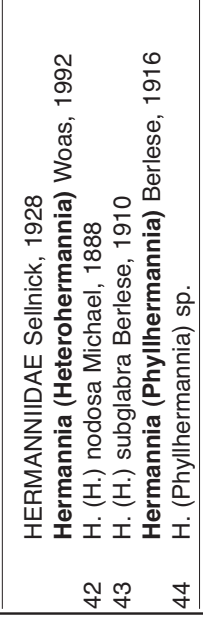 & 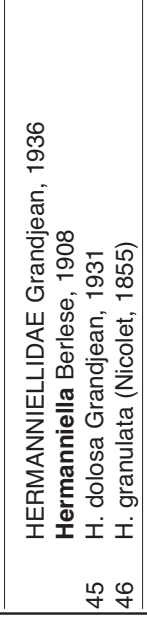 & 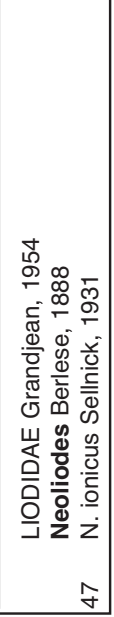 \\
\hline
\end{tabular}




\begin{tabular}{|c|c|c|c|c|c|c|c|c|c|c|c|c|}
\hline $\bar{\Sigma}$ & & & & & & & & & & & & \\
\hline$\uplus$ & & & $\times$ & & & $\times$ & & & $\times$ & & & \\
\hline 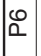 & & & & & $x$ & & & $\times$ & & $\times$ & & \\
\hline 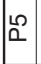 & & & & & $\times$ & $\times$ & & & & & $\times$ & \\
\hline$a$ & & & & & & & & & & & & \\
\hline 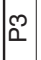 & & & & & & & & & & & & \\
\hline$\tilde{\alpha}$ & & & & & & & & & & & & \\
\hline $\bar{\Sigma}$ & & & & & & $\times \quad \times$ & & & & & & \\
\hline$\tilde{\delta}$ & & & & & & & & & & & & \\
\hline o & & & & & & & & & & & & \\
\hline$\tilde{~}$ & & & & & $\times$ & & & & & & & \\
\hline$\overline{0}$ & & & & & & & & & & & & \\
\hline$\Im$ & & & & & & & & & & & & \\
\hline$\Xi$ & & & & & & & & & & & & \\
\hline$\frac{\hat{z}}{z}$ & & & & & & & & & & & & \\
\hline 3 & & \& & & \& & \& & & & & & & & \\
\hline$\circ$ & वे & & $\begin{array}{ll}\dot{c} & 0 \\
\end{array}$ & & $\overline{0}$ & ஜ & ผ & & & & & \\
\hline$\tilde{o}$ & & & & & & & $\tilde{\omega}$ & & & & & \\
\hline 0 & 용 & & & & & & & : & & & & \\
\hline 3 & & & & & & & ๓ & & & & & \\
\hline 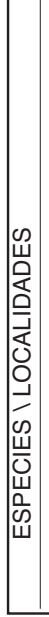 & 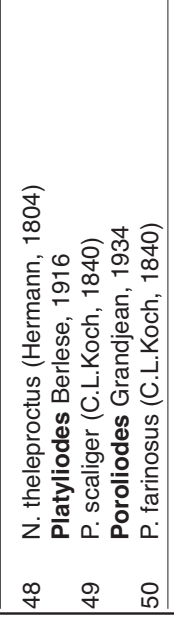 & 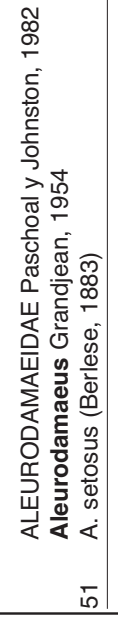 & 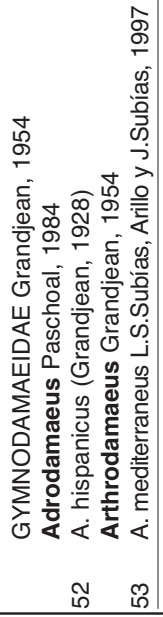 & 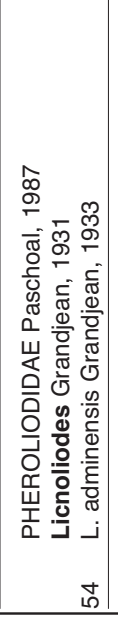 & 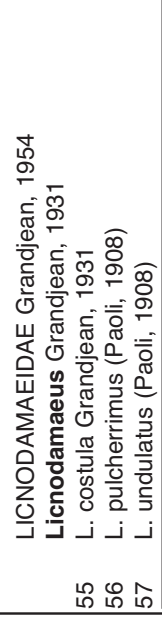 & 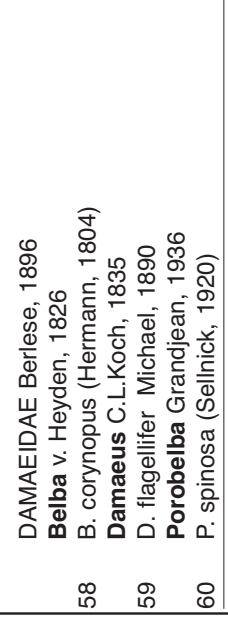 & 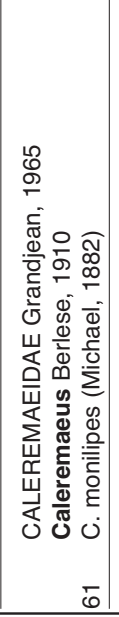 & 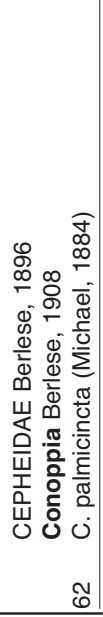 & 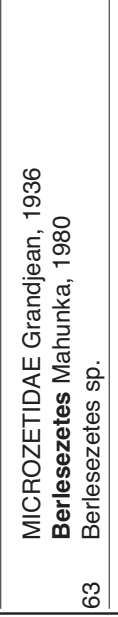 & 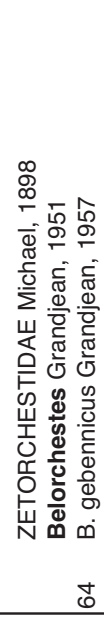 & 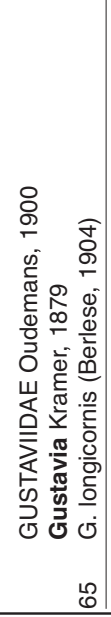 & 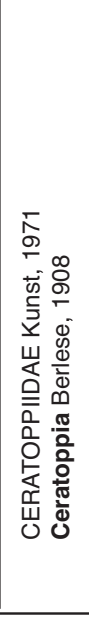 \\
\hline
\end{tabular}




\begin{tabular}{|c|c|c|c|c|c|c|c|c|c|c|c|}
\hline $\bar{\Sigma}$ & & & & & & & & & \multicolumn{2}{|r|}{$x$} & \\
\hline$\uplus$ & & $\times$ & & & & & & & & $\times$ & \\
\hline$\%$ & $\times$ & & $\times$ & & $\times$ & & & & $\times$ & $\times x \times x$ & \\
\hline 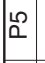 & $\times$ & & & & & & $\times$ & & & $\times \quad \times$ & \\
\hline$a^{a}$ & & & & & & & $\times \quad \times$ & & & & \\
\hline @ & & $\times$ & $\times$ & & & & & & & & \\
\hline$\Sigma$ & & & & & & & & & & & \\
\hline $\bar{a}$ & & $\times$ & & & & & & & & & \\
\hline$\tilde{\delta}$ & & & & & & & & & & & \\
\hline$\overline{0}$ & & & & & & & & & & & \\
\hline ชิ & & & & & & $\times$ & $\times$ & $\times$ & & & \\
\hline $\bar{j}$ & & & & & & & & & & & \\
\hline$\Im$ & & & & & & $\times$ & & & & & \\
\hline$=$ & & & & & & & & & & & \\
\hline$\hat{\mathbf{z}}$ & & & & & & & & & & & \\
\hline 3 & & & & & & & \& & & & \& & \\
\hline 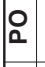 & $a$ & & & $\infty$ & $\stackrel{ \pm}{\Sigma}$ & a & & & $\tilde{\omega}$ & ஸ ळ & $\tilde{\omega}$ \\
\hline$\tilde{\alpha}$ & & & & & & & & & & ஸे $\tilde{\omega}$ & $\tilde{\omega}$ \\
\hline 0 & & & ○ & & & & & & & $\tilde{\omega}$ & \\
\hline 3 & & & & & & & & & & & \\
\hline 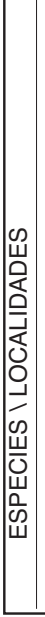 & 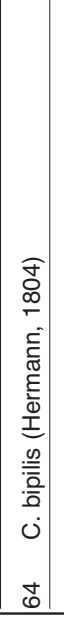 & 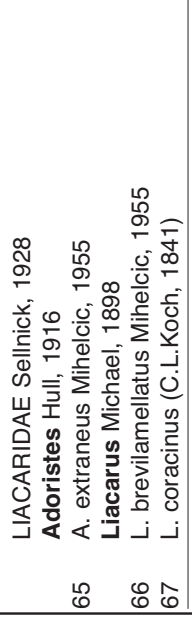 & 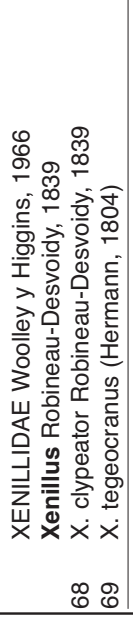 & 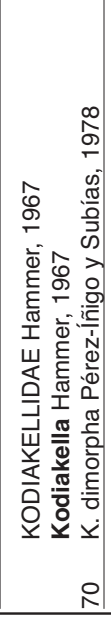 & 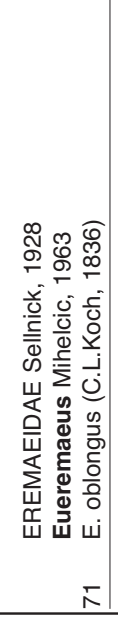 & 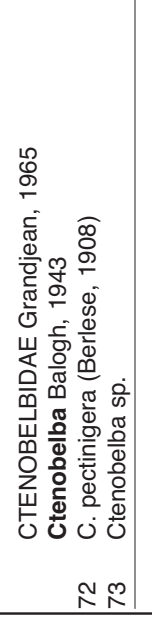 & 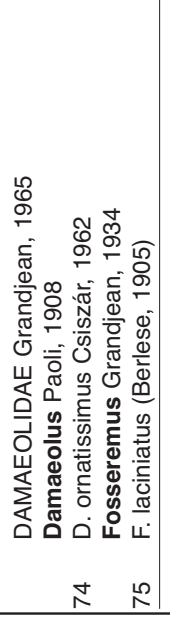 & 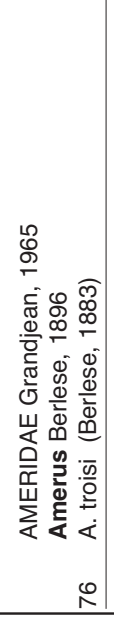 & 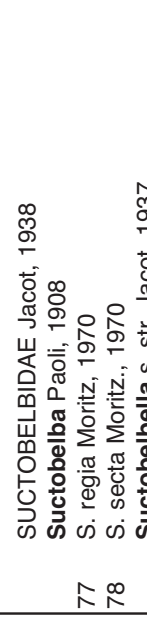 & 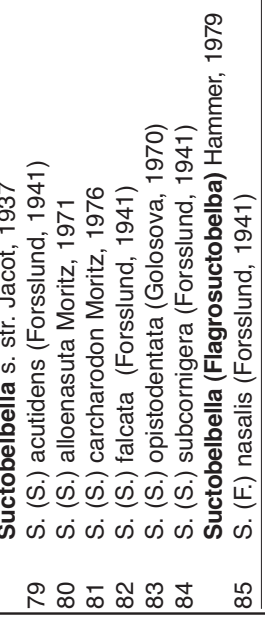 & 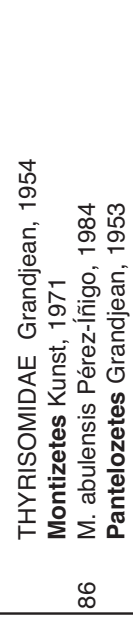 \\
\hline
\end{tabular}




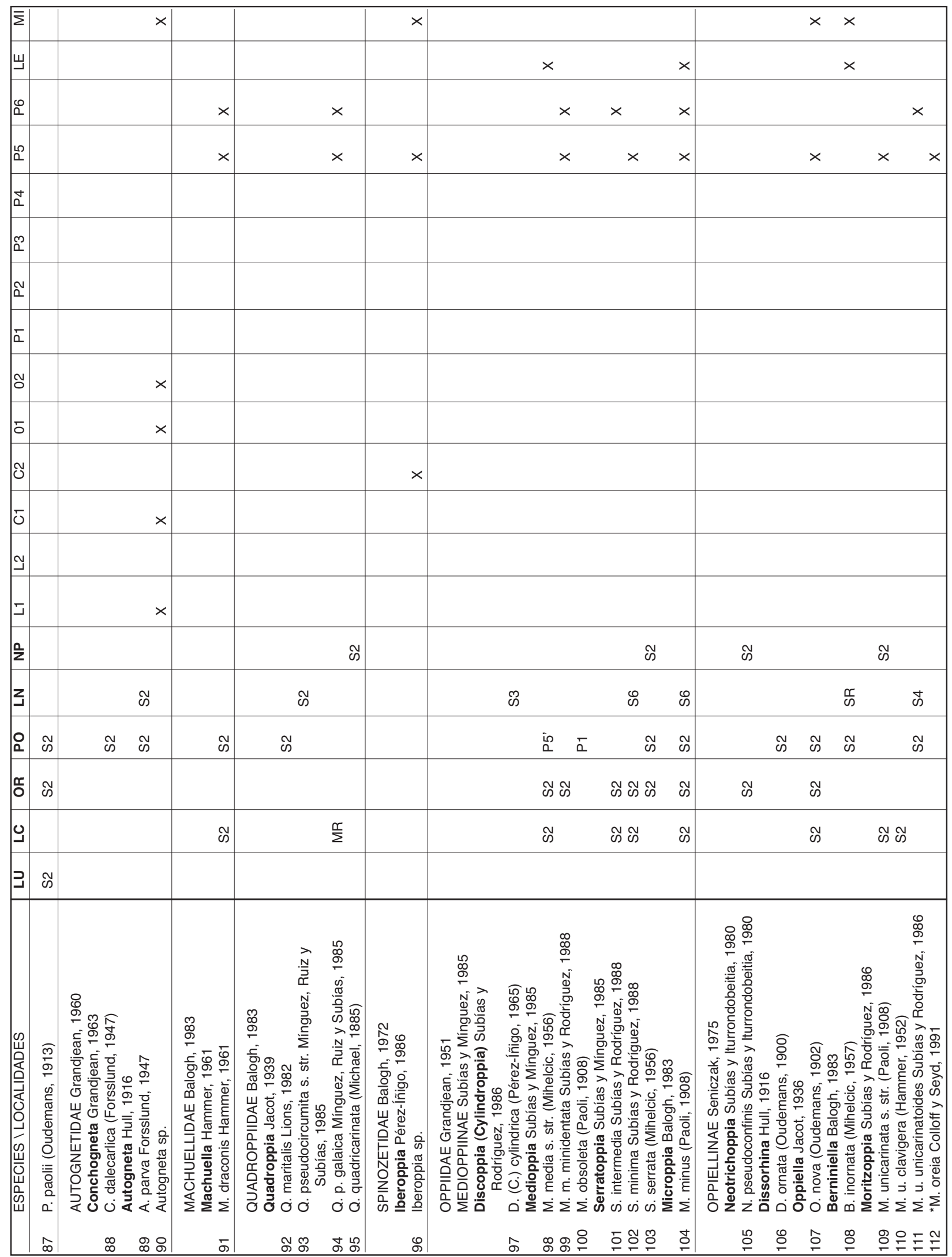




\begin{tabular}{|c|c|c|c|c|c|c|c|c|c|c|c|}
\hline $\bar{\Sigma}$ & & & & & & & 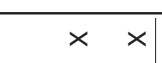 & $\times$ & & & \\
\hline$\uplus$ & & $\times$ & $\times$ & $\times$ & & & $\times$ & & & & \\
\hline 0 & & $\times$ & $\times$ & $\times \times x \quad \times$ & $\times$ & $\times$ & $\times$ & & & & \\
\hline & $\times$ & & $\times$ & $\times$ & $\times$ & & $\times$ & & & & \\
\hline$a$ & & & & & & $\times \times$ & & & & & \\
\hline$\infty$ & & & & & & & & & & & \\
\hline 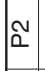 & & & & & & $\times$ & & & $\times$ & & \\
\hline $\bar{c}$ & & & & & & $\times \quad \times$ & $\times$ & & & & \\
\hline$\approx$ & & & & & & & & & & & \\
\hline 5 & & & & & & $\times$ & $\times$ & & & & \\
\hline ชิ & & & & & & $\times \quad \times$ & & & & & \\
\hline$\overline{0}$ & & & & & & & & & & & \\
\hline$\Im$ & & & & & & $\times \quad \times$ & & & & & \\
\hline$\Xi$ & & & & & & $x \times x \times \quad \times \quad \times$ & & & & & \\
\hline$\frac{\hat{z}}{\mathbf{z}}$ & & & & กิ & & & & & O & & \\
\hline 3 & & & ڤ & & & & & & & & \\
\hline : & $\Sigma$ & กิ & & ஸ & & & & & $\stackrel{\circ}{\circ}$ & $\underset{\circlearrowleft}{\infty}$ & \\
\hline$\tilde{\tilde{o}}$ & $\tilde{\infty}$ & ஸे స్ & & ஸ & & & & & & & \\
\hline 0 & & ผ & & ผ & & & & & & & \\
\hline 3 & & & & & & & & & & & \\
\hline 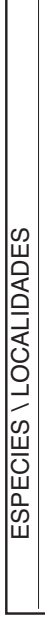 & 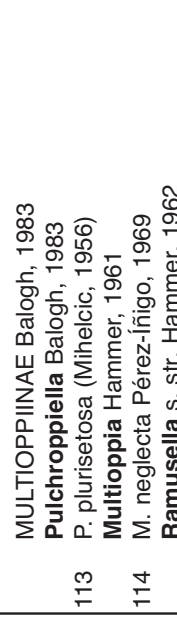 & 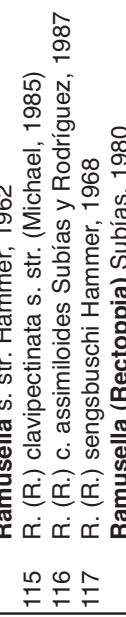 & 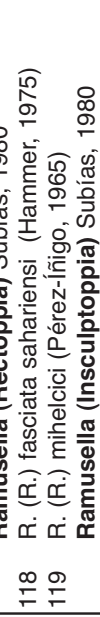 & 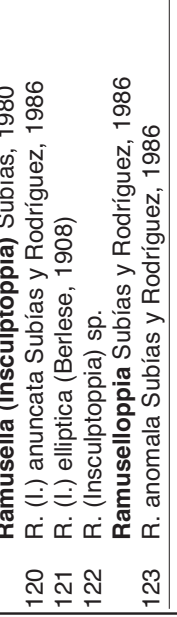 & 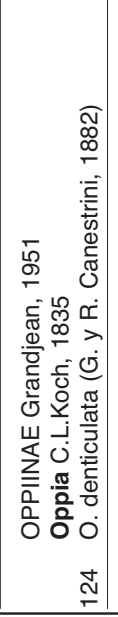 & 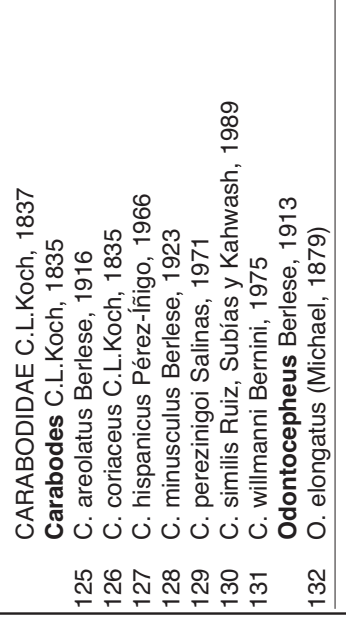 & 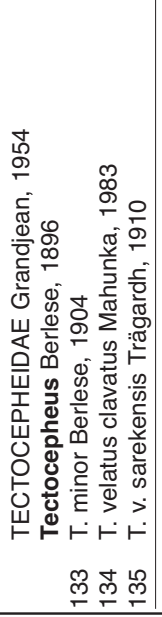 & 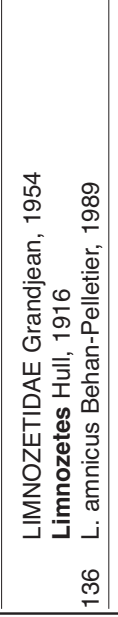 & 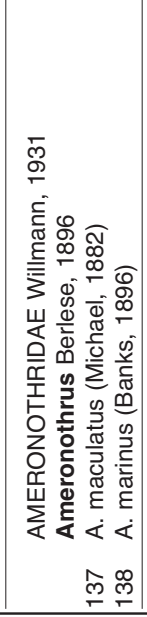 & 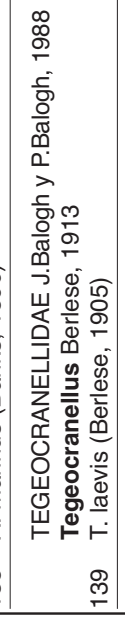 & 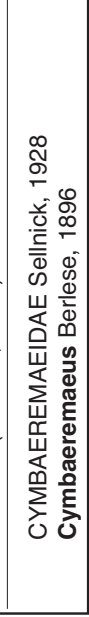 \\
\hline
\end{tabular}




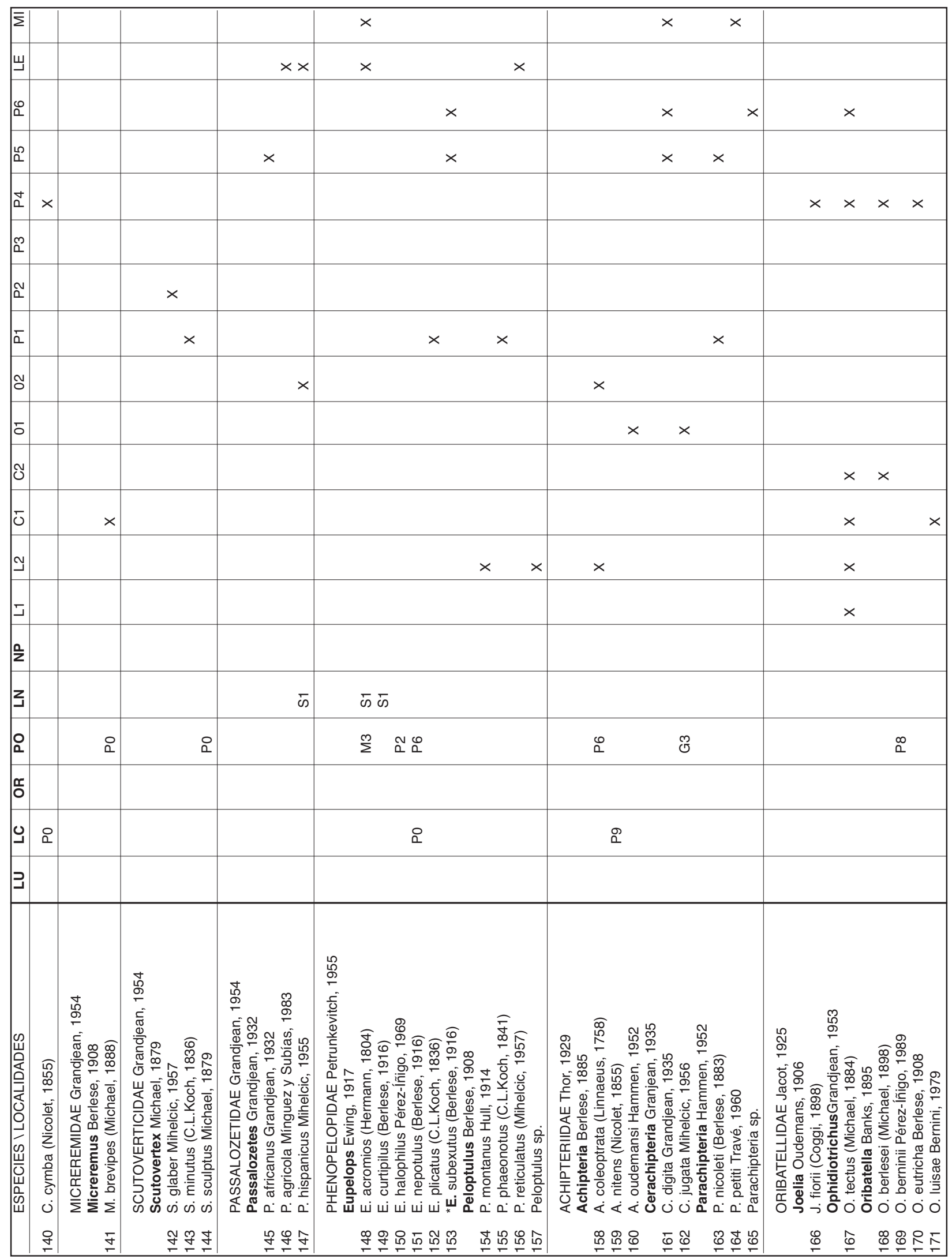




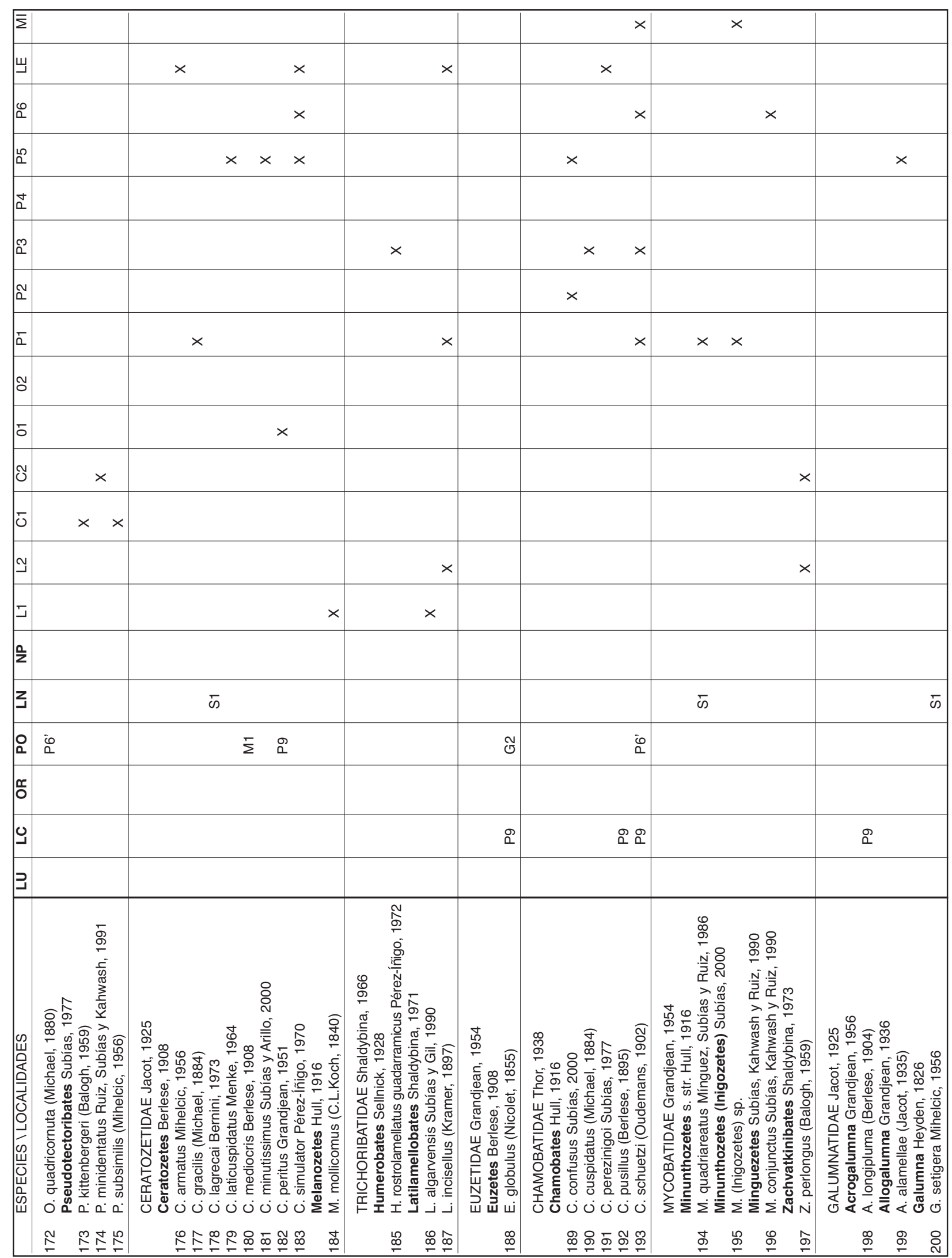




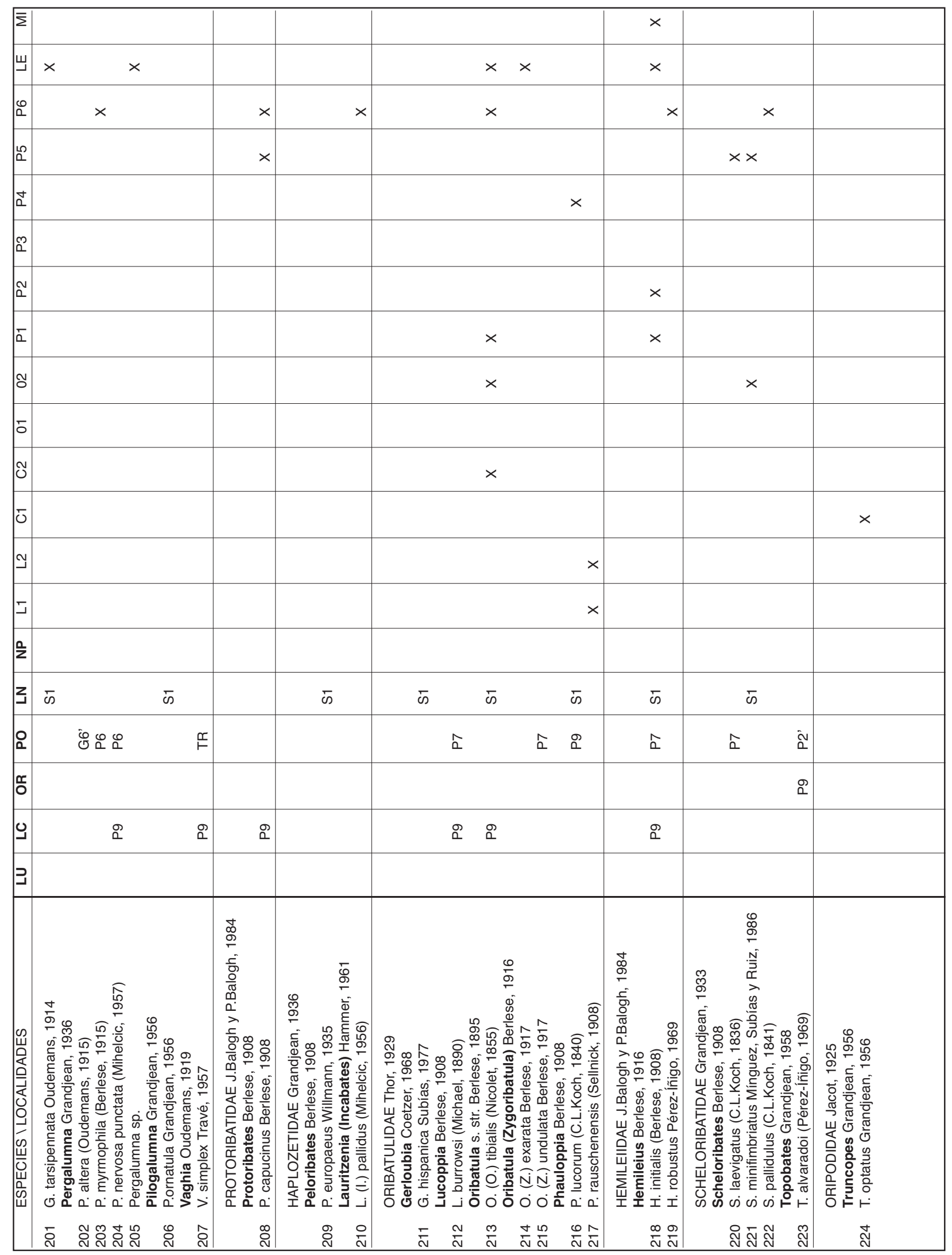




\section{Referencias}

Aoki, J., Yamamoto, Y., Wen, Z., Wang, H. \& Hu, S., 1997. [A Cheklist of Oribatid Mites of China (Acari: Oribatida). First Report.] Bulletin of the Institute of Environmental Sciencie and Technology, Yokohama National University, 23: 63-80. (En japonés).

Arribas, M.A., Subías, L.S. \& Ruiz, E., 1984. Oribátidos (Acarida, Oribatida) Superiores Gimnonóticos del "sabinar albar" español. Cuadernos de Investigación Biológica, 5: 57-63.

GRANDJEAN, F., 1928. Deux nouveaux Oribatei d'Espagne. Bulletin de la Société Zoologique de France, 53: 424-442.

Grandjean, F., 1931. Observations sur les Oribates (2 série). Bulletin du Muséum d'Histoire Naturelle, (2), 3: 651-665.

Grandjean, F., 1932. Au sujet des Palaeacariformes Trägardh. Bulletin du Muséum d'Histoire Naturelle, (2), 4: 411-426.

GRANDJEAN, F., 1935. Observartions sur les Oribates (9 série). Bulletin du Muséum d'Histoire Naturelle, (2), 7: 280-287.

GRANDJEAN, F., 1936. Les Oribates de Jean Frédéric Hermann et de son père. Annales de la Société Entomologique de France, 105: 27-110.

Grandjean, F., Observations sur les Oribates (30 série). Bulletin du Muséum d'Histoire Naturelle, (2), 26: 482-490.

GRANDJEAN, F., 1956a. Observations sur les Galumnidae (1 série) (Acariens, Oribates). Revue Française d'Entomologie, 23: 137-146.

GrandJEAn, F., 1956b. Observations sur les Galumnidae (2 série) (Acariens, Oribates). Revue Française d'Entomologie, 23: 265-275.

GrandJEAN, F., 1962. Le genre Tegeocranellus Berl., 1913 (Oribates). Acarologia, 4: 78-100.

Minelcic, F., 1956a. Oribatiden Südeuropas IV. Zoologischer Anzeiger, 156: 205-226.

Minelcic, F., 1956b. Oribatiden Südeuropas V. Zoologischer Anzeiger, 157: 154-174.

Minelcic, F., 1957. Oribatiden der iberischen Halbinsel VI. Zoologischer Anzeiger, 158: 53-66.

Minelcic, F., 1964. Contribución al conocimiento de la ecología y la distribución geográfica del género Eremaeus C. L. Koch. Anales de Edafología y Agrobiología, 23: 547-552.

Mínguez, M.E., RuIz, E. \& SubíAs, L.S., 1985. El género Quadroppia Jacot, 1939 (Acari, Oribatida, Oppiidae). Boletín de la Asociación Española de Entomología, 9: 95-118.

Mínguez, M.E. \& SubÍAS, L.S., 1998. Confirmacion de la presencia de Trhypochthoniellus excavatus (Willmann, 1919) en la Península Ibérica (Acari: Oribati- da: Trhypochthoniidae). Boletín de la Asociación Española de Entomología, 22: 242.

NiedBALA, W., 1984a. Mesoplophoroidea (Acari, Oribatida). Changement du système et redescription d'espèces-types. Bulletin de l'Académie Polonaise des Sciences, Série des Sciences Biologiques, 32: 137-155.

NiedBALA, W., 1984b. Deux nouveaux Phthiracaridae (Acari, Oribatida) d'Espagne. Bulletin de l'Académie Polonaise des Sciences, Série des Sciences Biologiques, 32: 267-273.

Niedbala, W., 1992. Phthiracaroidea (Acari, Oribatida). Systematic Studies. PWN - Elsevier. 612 pp.

PÉREZ-ÍñIgO, C., 1965. Especies españolas del género Oppia C. L. Koch (Acari, Oribatei). Boletín de la Real Sociedad Española de Historia Natural (Sección Biológica), [1964], 62: 385-416.

PÉrez-ÍñIgo, C., 1969a. Nuevos oribátidos de suelos españoles (Acari, Oribatei). Eos, [1968], 44: $377-$ 403.

PÉrez-ÍÑIGO, C., 1969b. Ácaros oribátidos de suelos de España Peninsular e Islas Baleares ( $1^{\mathrm{a}}$ Parte) (Acari, Oribatei). Graellsia, [1968], 24: 143-238.

PÉreZ-ÍÑ̃Go, C., 1970. Ácaros oribátidos de suelos de España Peninsular e Islas Baleares (Acari, Oribatei). Parte II. Eos, [1969], 45: 241-317.

PÉREZ-ÍÑIGO, C., 1971.Ácaros oribátidos de España Peninsular e Islas Baleares (Acari, Oribatei). Parte III. Eos, [1970], 46: 263-349.

PÉrez-ÍÑigo, C., 1972. Ácaros oribátidos de suelos de España Peninsular e Islas Baleares (Acari, Oribatei). Parte IV. Eos, [1971], 47: 247-333.

PÉrez-ÍÑigo, C., 1974. Ácaros oribátidos de suelos de España Peninsular e Islas Baleares (Acari, Oribatei). Parte V. Eos, [1972], 48: 367-475.

PÉrez-Í̃̃̃go, C., 1988. Catálogo de Oribátidos (Acari, Oribatei) de la Sierra de Guadarrama. Graellsia, 44: 31-64.

PéReZ-ÍñIgO, C., 1993. Acari. Oribatei, Poronota. En: Fauna Ibérica, vol. 3. Ramos, M.A. et al. (Eds.). Museo Nacional de Ciencias Naturales, CSIC. Madrid. 320 pp.

PÉrEz-ÍÑIGO, C., 1997. Acari. Oribatei, Gymnonota I. En: Fauna Ibérica, vol. 9. Ramos, M.A. et al. (Eds.). Museo Nacional de Ciencias Naturales. Madrid. 373 pp.

PÉREZ-ÍÑIGO, C. \& SubÍAS, L.S., 1978. Sorprendente hallazgo de un representante de la familia Kodiakellidaee Hammer en España, Kodiakella dimorpha n. sp., y consideraciones sobre esta familia (Acari, Oribatei). Boletín de la Asociación Española de Entomología, [1977], 1: 103-107.

RuIz, E. \& SubÍAS, L.S., 1984. Oribátidos (Acarida, Oribatida) Inferiores del "sabinar albar" español. Boletín de la Asociación Española de Entomología, 8: 195201. 
SCHUBART, H., 1975. Morphologische Grundlagen für die Klärung der Verwandtschaftsbeziehungen innerhalb der Milbenfamilie Ameronothridae (Acari). Zoologica, 123: 23-91.

SuBíAs, L.S., 1982. Oribátidos de Murcia I. (Oribátidos Inferiores. Parte I) (Acarida, Oribatida). Anales de la Universidad de Murcia, [1979-80], 38: 133-151.

SubíAs, L.S. \& ArILlo, A., 1998. Oribátidos (Acari, Oribatida) superiores poronóticos del sabinar albar (Juniperus thurifera) español. Listado sistemático, descripción de Zygoribatula hispanica sp. nov. y consideraciones biológicas. Boletín de la Asociación Española de Entomología, 22: 63-74.

Subías, L.S. \& Arillo, A., (En prensa). Acari. Oribatei, Gymnonota II, Oppioidea. En: Fauna Ibérica, vol. 15. Ramos, M.A. et al. (Eds.). Museo Nacional de Ciencias Naturales, CSIC. Madrid.

SubíAs, L.S. \& Rodríguez, P., 1986a. Oppiidae (Acari, Oribatida) de los sabinares (Juniperus thurifera) de España IX. Subiasella (Lalmoppia) n. subgen. y Discoppia (Cylindroppia) n. subgen. Revista de Biología de la Universidad de Oviedo, 4: 111-121.

Subías, L.S. \& Rodríguez, P., 1986b. Oppiidae (Acari, Oribatida) de los sabinares (Juniperus thurifera) de España VI. Neotrichoppia (Confinoppia) n. subg. y Moritziella Balogh, 1983. Redia, 69: 115-130.

Subías, L.S. \& Rodríguez, P., 1987. Oppiidae (Acari, Oribatida) de los sabinares (Juniperus thurifera) de España I. Ramusella s. str. Hammer y Ramusella (Rectoppia) Subías. Eos, 63: 301-314.
Subías, L.S. \& RodríguEz, P., 1988. Oppiidae (Acari, Oribatida) de los sabinares (Juniperus thurifera) de España VIII. Medioppiinae Subías y Mínguez. Boletín de la Asociación Española de Entomología, 12: 27-43.

Subías, L.S., Rodríguez, P. \& Mínguez, M.E., 1987. Los Oppiidae (Acari, Oribatida) de los sabinares (Juniperus thurifera) de España, V. Berniniella Balogh, 1983. Cuadernos de Investigación Biológica, 10: $35-50$

Travé, J., 1957. Compléments à la connaissance du genre Vaghia Oudemans. Vaghia simplex n. sp. (Acariens, Oribates). Vie et Milieu, 8: 205-210.

WOAS, S., 1981. Zur Taxonomie und Phylogenie der Hermanniidae Sellnick, 1928 (Acari, Oribatei). Andrias, 1: 7-88.
Recibido, el 28-XI-2000

Aceptado, el 19-IV-2001 Publicado, el 00-XX-2001 